\title{
GAS FEEDING TOWARD THE CENTRAL NEUTRAL RING IN THE GALACTIC CENTER
}

\author{
P. T. P. HO ${ }^{1}$, J. M. JACKSON ${ }^{2}$, J. T. ARMSTRONG ${ }^{3}$ \\ and J. C. SZCZEPANSKI, ${ }^{1,4}$ \\ 1 Harvard-Smithsonian Center for Astrophysics, Cambridge,MA \\ 2 University of California, Berkeley, $C A$ \\ 3 Universität zu Köln, Köln, FRG \\ Massachusetts Institute of Technology, Cambridge, MA
}

\begin{abstract}
VLA observations in the $(\mathrm{J}, \mathrm{K})=(3,3)$ line of ammonia reveal new structures in the Galactic center region. An approximate ring of emission is centered on the central ionized streamers. This ring, seen previously in millimeter-wave interferometer maps, is very clumpy in the ammonia emission, with size scales $\leqslant 10^{\prime \prime}(0.4 \mathrm{pc})$. The clumps show good spatial and velocity agreement with the ionized gas, and are warm with brightness temperatures exceeding $30 \mathrm{~K}$. A comparison of the $(3,3)$ to $(1,1)$ ratio indicates considerably higher gas temperatures. This circumnuclear ring may not be the dominant feature in the mass distribution of the circumnuclear gas. A streamer, immediately to the south of the Galactic center, connects the gas complex at $l_{I I}=-4^{\prime}(\sim 10 \mathrm{pc})$ directly to the Galactic center. This streamer may define the path for gas flow into the nuclear region.
\end{abstract}

\section{Introduction}

The Galactic center is observed in the $(\mathrm{J}, \mathrm{K})=(3,3)$ line of $\mathrm{NH}_{3}$ using the VLA (Figure 1 ). $A$ number of emission features are seen with $10^{\prime \prime}$ resolution, which are external to the $2 \mathrm{pc}$ circumnuclear ring (Genzel and Townes 1987; Güsten et al. 1987). The north-south ionized streamer (Lo and Claussen 1983) (our 1-cm map is shown in Figure 2) is closely associated with a similar structure in $\mathrm{NH}_{3}$. However, the east-west ionized streamer is associated with very faint emission which may have originated in an eastern $\mathrm{NH}_{3}$ feature $\sim 1^{\prime}$ from the center. There is a western $\mathrm{NH}_{3}$ feature which is also $\sim \mathbf{1}^{\prime}$ from the Galactic center, while a southern arm apparently connects the central region to the giant molecular cloud $\mathrm{M}-0.13-0.08$.

\section{The $\mathrm{NH}_{3}$ Streamers}

The $(\mathrm{J}, \mathrm{K})=(3,3)$ line of $\mathrm{NH}_{3}$ is sensitive to the hotter gas (100 K above ground). Its emission is not contaminated by cold and optically thick emission structures along the line of sight, as in the case of the HCN observations (Güsten et al. 1987). In particular, we are not sensitive to the high velocity gas that is most evident in CO observations (Serabyn et al. 1986). We conclude that we are picking out gas features local ( $\leqslant 3 \mathrm{pc})$ to the Galactic center. The $\mathrm{NH}_{3}$ streamers are evident in the total intensity map (Fig. 1). Compared to the map of 1-cm continuum emission (Fig. 2), we can see that there are three new 


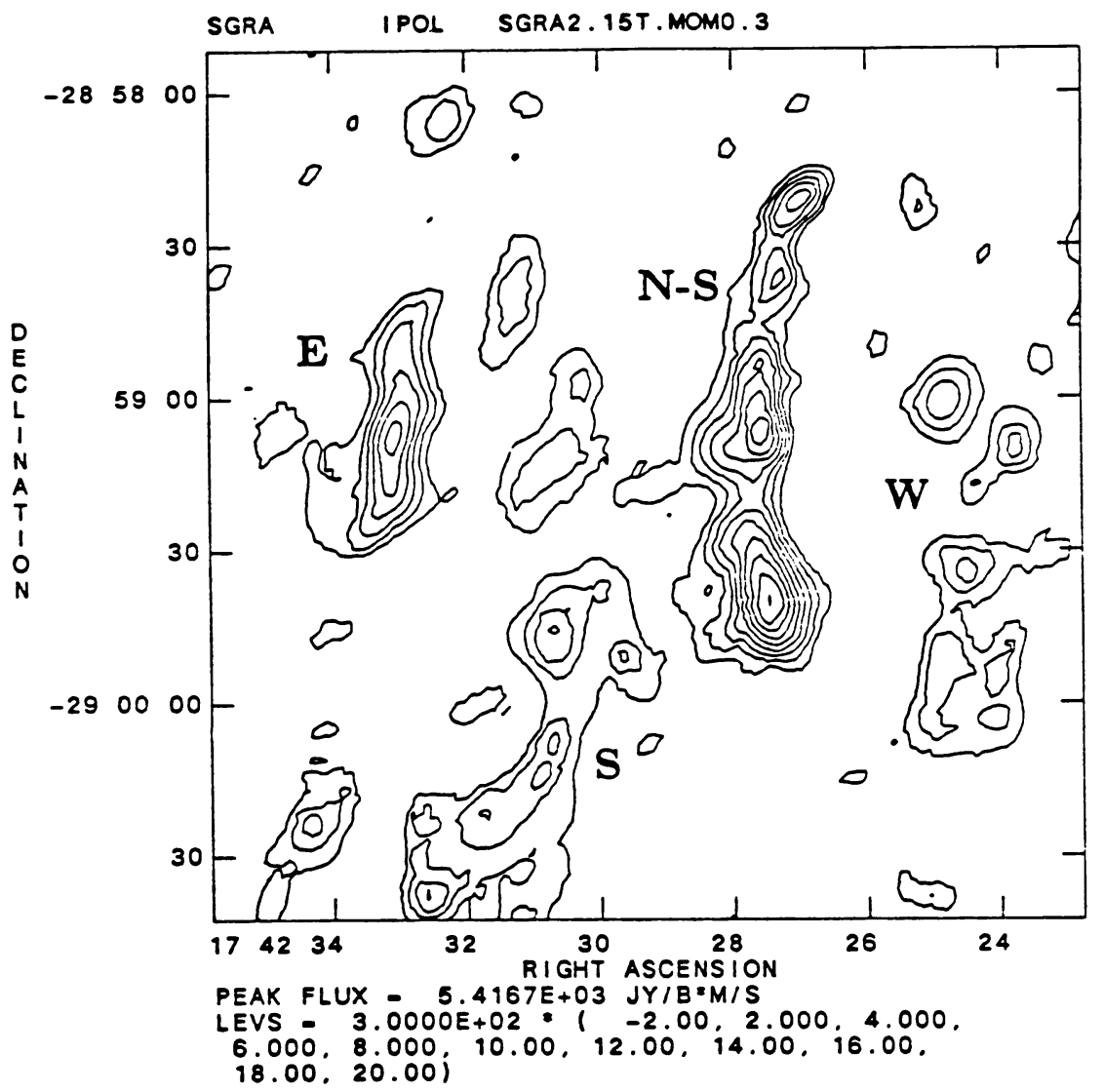

Figure 1. Integrated intensity in the $(3,3) \mathrm{NH}_{3}$ line.

significant features outside of the circumnuclear ring: an eastern (E) feature, a western (W) feature, and a southern (S) feature. Position-velocity diagrams show that these features are cohesive both in space and velocity. An east-west cut along the east-west ionized streamer suggests that neutral gas along this ionized streamer may have originated from the " $E$ " $\mathrm{NH}_{3}$ feature. $\mathrm{NH}_{3}$ observations have also been obtained in the adjacent VLA primary beam to the south. The " $\mathrm{S}$ " feature can be seen to continue toward the giant molecular cloud located at $\sim 5^{\prime}$ south of the Galactic center. In the high sensitivity $90-\mathrm{cm}$ continuum maps shown by Pedlar et al. (1988), the north-south ionized streamer, seen in absorption, extends to the south in the direction of the $\mathrm{NH}_{3}$ "S" streamer. This streamer may be the path by which gas is fed toward the Galactic center. In other papers presented at this meeting (Okumura et al.; Szczepanski et al.), evidence is presented which shows that the impact of a possible SNR against the molecular cloud $\mathrm{M}-0.13-0.08$ south of the Galactic center may be driving gas toward the center in the form of the " $\mathrm{S}$ " streamer. 


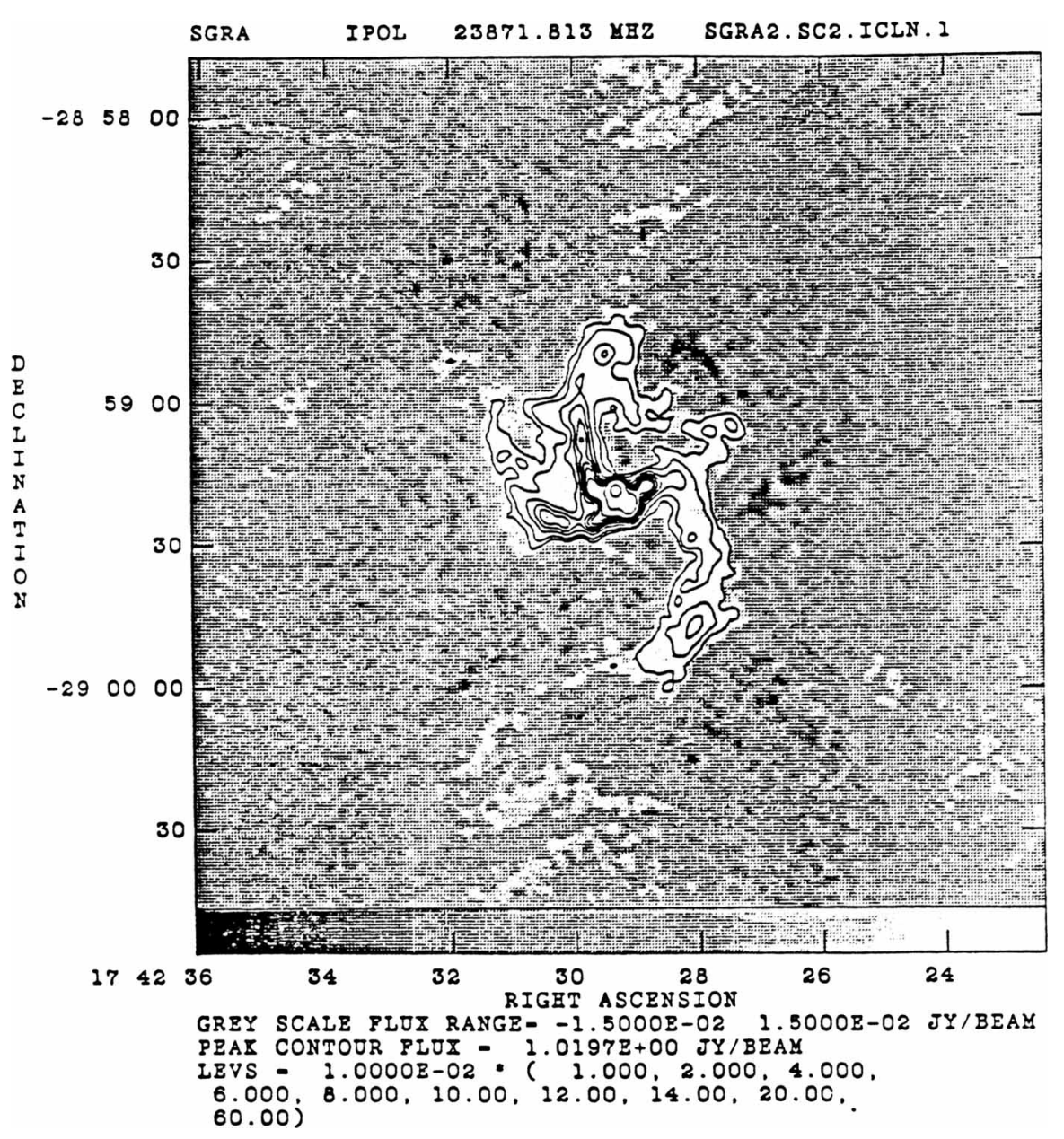

Figure 2. 1-cm continuum emission.

\section{Circumnuclear Disk}

The circumnuclear ring is extremely clumped and does not resemble a complete structure. The observed $\mathrm{NH}_{3}$ features suggest that gas is fed toward the center from nearby giant molecular clouds in a most nonuniform manner. Both line widths and relative motions become large in magnitude only at $\sim 1^{\prime}$ from the Galactic center. These clumps, though not a cohesive shell or disk, are probably the origin of the observed ionized streamers. Although these results and the $\mathrm{HCN}$ results agree in general, there are significant differences that are quite disturbing. One possibility is optical depth and contamination by low excitation gas along the line of sight. Another possibility is to appeal to chemistry. Whatever the detailed 
structures of the circumnuclear gas, the most important point here is that gas appears to be fed into the Galactic center via a thin streamer from the south.

\section{References}

Genzel, R., and Townes, C. H. 1987, Ann. Rev. Astr. Ap., 25, 377.

Güsten, R., Genzel, R., Wright, M. C. H., Jaffee, D. T., Stutzki, J., and Harris, A. I. 1987, Ap. J., 318, 124.

Lo, K. Y., and Claussen, M. J. 1983, Nature, 306, 647.

Okumura-Kawabe, S., Ishiguro, M., Fomalont, E. B., Chikada, T., Kasuga, T., Morita, K. -I., Kawabe, R., Kobayashi, H., Hasegawa, T., and Inoue, M. these proceedings.

Pedlar, A., Anantharamaiah, K. R., Ekers, R. D., Goss, W. M., van Gorkom, J. H., Schwarz, U. J., and Zhao, J. these proceedings.

Serabyn, E., Güsten, R., Walmsley, C. M., Wink, J. E., and Zylka, R. 1986, Astr. Ap., 169, 85.

Szczepanski, J. C., Ho, P. T. P., Haschick, A. D., and Baan, W. A. these proceedings. 